\title{
Residential Demand Management using Individualised Demand Aware Price Policies
}

\author{
Barry Hayes, Member, IEEE, Igor Melatti, Toni Mancini, \\ Milan Prodanovic, Member, IEEE, and Enrico Tronci.
}

\begin{abstract}
This paper presents a novel approach to Demand Side Management (DSM), using an "individualised" price policy, where each end user receives a separate electricity pricing scheme designed to incentivise demand management in order to optimally manage flexible demands. These pricing schemes have the objective of reducing the peaks in overall system demand in such a way that the average electricity price each individual user receives is non-discriminatory. It is shown in the paper that this approach has a number of advantages and benefits compared to traditional DSM approaches. The "demand aware price policy" approach outlined in this paper exploits the knowledge, or demand-awareness, obtained from advanced metering infrastructure. The presented analysis includes a detailed case study of an existing European distribution network where DSM trial data was available from the residential end-users.
\end{abstract}

\section{NOMENCLATURE}

Indices and sets

$S$

$s$

$T$

$t$

$U$

$u$

$\mathcal{T}$

$\mathcal{P}$

set of substation indices

indices of substations (elements of $S$ )

set of time-slots indices

indices of time-slots (elements of $T$ )

set of residential user indices

indices of residential users (elements of $U$ )

tariff system

set of individualised price policies

\section{Parameters and Constants}

$\begin{array}{ll}d_{u}^{t} & \begin{array}{l}\text { forecasted power demand of user } u \text { in time-slot } t \\ \tilde{d}_{u}^{t}\end{array} \\ \hat{d}_{u}^{t} & \begin{array}{l}\text { historical power demand of user } u \text { in time-slot } t \\ \text { power demand of user } u \text { in time-slot } t, \text { as a response } \\ \text { to individualised price policies }\end{array} \\ P_{S}^{t} & \begin{array}{l}\text { maximum power for substation } s \text { in time-slot } t \\ Q_{u}\end{array} \\ R_{u} & \text { capacity of storage of user } u \\ C_{u} & \text { power rate of storage of user } u \\ \alpha & \text { maximum power for user } u, \text { from energy contract } \\ & \begin{array}{l}\text { low-tariff energy } \\ b_{l}\end{array} \quad \begin{array}{l}\text { residential users low price for buying energy } \\ b_{h}\end{array} \quad \begin{array}{l}\text { residential users high price for buying energy } \\ \beta\end{array} \quad \begin{array}{l}\text { residential users price for selling energy from local } \\ \text { renewable sources }\end{array}\end{array}$

The authors are with the IMDEA Energy Institute, Avda. Ramón de la Sagra, Móstoles Technology Park, Madrid 28935, Spain, and Sapienza University of Rome, via Salaria 113, 00198 Rome, Italy. Corresponding author e-mail: barry.hayes@imdea.org. The research leading to these results has received funding from the European Union Seventh Framework Programme (FP7/2007-2013) under grant agreement number 317761 (SmartHG) and the Spanish Ministry of Economy and Competitiveness project RESmart (ENE2013-48690-C2-2-R).

\section{Variables}

$b_{u}^{t} \quad$ state of charge of storage of user $u$ in time-slot $t$

$a_{u}^{t} \quad$ charge/discharge action on storage of user $u$ in timeslot $t$

$\Delta^{t} \quad$ exceeding power at substation level

$P_{u}^{t} \quad$ maximum power for user $u$ in time-slot $t$

$\zeta_{u} \quad$ percentage of load shifting required for user $u$

$\xi \quad$ index of price policy non-discrimination

\section{INTRODUCTION}

$\mathbf{T}$ His paper presents a set of Demand Side Management (DSM) software services which were introduced in the SmartHG project [1]. These services are designed to manage residential end-user energy demand with two objectives: to minimize energy costs for each individual user, and to assist the Distribution System Operator (DSO) in managing network constraints and optimising the operation of the distribution system. This is achieved by exploiting demand awareness as obtained from smart metering and other Advanced Metering Infrastructure (AMI). One of the unique aspects of the approach introduced in the SmartHG project is that the price policies are "individualised", e.g. each individual user receives a separate electricity pricing scheme designed to incetivise demand management in order to optimally manage flexible demands. These pricing schemes are designed with the primary objective of reducing the peaks in overall distribution system demand, which has significant benefits for the network and for the DSO. This is achieved in such a way that the average electricity price each individual user receives is nondiscriminatory, and the pricing policy is designed to shape the demand without reducing the overall demand volume, which is undesirable from the DSO point of view.

The paper is structured as follows: Section II discusses previous work, Section III contains the problem formulation, and Section IV outlines the methodology. Section V describes the case study and results, and Section VI concludes.

\section{Previous Work}

In light of the increasing penetrations of variable energy resources, and the decreasing contribution from traditional generation sources, i.e. large, controllable thermal generation plant, a number of recent studies have identified a need for new sources of flexibility in electricity networks, e.g., [2], [3]. For several decades, network operators have used various forms of DSM to improve the balancing of system supply and demand and to reduce load peaks. Many of the practical DSM 
programmes implemented worldwide to date have focused on large industrial consumers since these have demand of sufficient volume to produce significant effects at the system level (e.g., [4], [5]). However, with the introduction of smart metering and time-varying electricity rates for individual customers, new opportunities are being created for small electricity users to participate in demand side services. Recently, much research has focused on DSM in the residential sector [6]-[13].

Many existing DSM programmes use direct load control, where the network operator is able to directly actuate large industrial loads according to the needs of the network [4], [5], and the exact terms of the DSM contract are agreed beforehand. While direct load control may be suitable for certain industrial users, it has technical and practical difficulties in the context of residential users, where direct actuation of loads is typically considered an invasion of user privacy and comfort. In addition, direct load control may require large investments in order to provide additional communication and control technology for each user. Most residential DSM schemes instead rely on the user response to a electricity price signal to produce the required outcome, e.g., [6], [7], [9]-[14].

Recent studies carried out in Ireland [15] and in Denmark [16], have tested the response of residential users to various Time of Usage (ToU) electricity pricing schemes, in order to quantify their potential to offer DSM services. It was shown in these trials that a significant amount of the residential demand (up to 19\% in [16]) could be shifted away from the peak hour if appropriate economic incentives are applied. In order to achieve the volumes of demand required to participate in the electricity market, and to make a significant contribution to system-level energy balancing, a means of combining and coordinating DSM actions from many highlydistributed users is required. Several approaches for this have been proposed, such as the "aggregator" [17] and "virtual power plant" concepts [18], [19].

One of the drawbacks of traditional approaches to DSM, where all users are subject to the same price (global price policy), is that the peak-shifting schemes may result in undesirable "rebound" effects, e.g. simply shifting the demand peaks from the peak hour to the off-peak hours, and creating new demand peaks, or "rebound peaks" [11]-[13]. The authors in [11] and [12] discuss automated DSM algorithms designed to schedule flexible residential user loads. It is demonstrated that this can produce rebound peaks, and it is concluded in [11] that DSM algorithms need to be studied for large numbers of devices and users, and that DSM schemes need to be designed with the objective of flattening the overall electricity usage in order to avoid these issues.

Exposing electricity end users to wholesale market prices (real-time pricing) has the drawback that demand may be shifted to hours with low electricity price, which can "lead to a higher peak electricity price and peak-to-average ratio during the low price time", according to [13]. There is a significant challenge in ensuring that such real-time prices do not cause physical or market instabilities [20], and it has been shown that multiple, uncoordinated responses to frequently changing prices can cause increased volatility, and potentially grid instability [21]-[23].

\section{PRoblem Formulation}

\section{A. Individualised User Price Policies}

A real-world example of the rebound effect is shown below, using recorded data from a residential DSM study carried out by a Danish DSO [16]. In this study, residential users were given a time-varying price policy with three distinct pricing periods, designed to test the flexibility of residential demand to economic peak-shifting incentives. The values are provided below in Danish Krone (DKK):

- Day: 1.50 DKK / kWh (06:00-17:00)

- Peak: 8.00 DKK / kWh (17:00-20:00)

- Night: $0.00 \mathrm{DKK} / \mathrm{kWh}$ (20:00-06:00)

This electricity pricing scheme provided strong incentives for household users and assessed the potential for residential users to shift their demand away from the peak cooking hours (17:00-20:00) to the night period (20:00-06:00) where the price is zero ${ }^{1}$.

The above price policy was applied to a "Test group" of 350 households, with a "Reference group" of 349 households ${ }^{2}$ receiving a fixed price of $2.25 \mathrm{DKK} / \mathrm{kWh}$ at all hours during the day (as per the standard flat tariff residential pricing scheme used in Denmark). The study was carried out over a full year from 1 October 2013 to 30 September 2014. The results showed that, with the above price incentives, a significant amount of residential demand (up to 19\%) was shifted away from the peak hours, compared to the reference group. The price incentives had little effect on the overall demand consumption, i.e. the total volume of consumption remained almost constant, only the times at which consumption occurred were influenced by the pricing scheme.

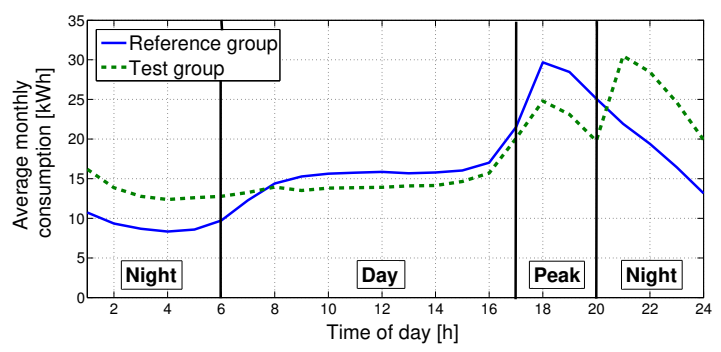

Fig. 1. Sample of the results from the Danish study, showing peak shifting in the Test group compared to the Reference group during January 2014 [16].

Fig. 1 shows a sample of the results from the Danish study [16] for the month January 2014. All months of the year showed a similar pattern, but the amount of demand shift from the peak hour was largest during the winter months.

The results in Fig. 1 are a good example of the "rebound peak" effects which can result from global ToU price policies.

\footnotetext{
${ }^{1}$ It could be argued that this is a rather extreme pricing policy and that the zero night time price is more likely to cause rebound peaks than a pricing scheme with, for example, a non-zero night-time price. However, the results of this study are insightful in that they demonstrate that significant residential demand-shifting is possible with appropriate financial incentives, and these results provide real data on user responses to price signals for the subsequent analysis in the paper.

${ }^{2}$ The SEAS-NVE study was originally designed with 350 test and 350 reference householders, but data was unavailable for one of the reference group households; hence this group only has 349 households.
} 
There is a significant increase in demand at the beginning of the off-peak period for the "Test group", and this pricing scheme has created a new demand peak at 21:00, which is even larger than the demand peak in the "Reference group", Fig. 1. These effects are undesirable, since typical DSM objectives are to smooth the load profile, increase the load factor, and reduce demand peaks.

An alternative solution to the "global" price policy, where all users (or at least all users in the same sector), receive the same price incentives is proposed in this paper. Instead, an "individualised" price policy is provided to each user, using the Demand-Aware Price Policy (DAPP) computational service outlined in Section IV. This is designed to maximise the benefits of demand response actions for both the user and the DSO. The effects on network operation and the potential benefits to the DSO in using such an individualised price policy are evaluated using the scenarios presented in Section V.

Even with strong ToU price incentives for demand-shifting, such as those used in the SEAS-NVE study [16]), the amount of demand flexibility from residential appliances is limited. Energy Storage Systems (ESS) offer much greater possibilities for demand flexibility. It is widely expected that the cost of such technologies will continue to decrease, making storage accessible to a wide range of users, including domestic consumers. Moreover, Plug-in Electric Vehicles (PEVs) can significantly alter the demand profiles, and create critical congestions in the distribution network if their charging is not managed appropriately [24], [25]. Accordingly, this paper also analyses scenarios where the residential homes are equipped with PEVs and ESS, in order to examine potential future scenarios with greater user flexibility in response to ToU pricing.

\section{Methodology}

\section{A. Overview}

The proposed methodology is based on two integrated software services, which are described schematically in Fig. 2. The Electricity Distribution Network Virtual Tomography (EVT) service is aimed at assisting the DSO in the operation and management of the distribution networks. The EVT service uses available measurements from Supervisory Control And Data Acquisition (SCADA) and smart metering/AMI systems to estimate the network state in real-time using a Weighted Least Squares (WLS) state estimator, and also to carry out network analysis ahead of time, as described in [26], [27]. This detects possible violations of network constraints, and raises warnings and alarms to the DSO accordingly. The results of the state estimation and network analysis carried out in the EVT can be used to make operational constraints, limiting the demand drawn at some or all substations $s$ within the distribution network at times of peak demand. This could be motivated by economic reasons (e.g. in order to reduce the cost for the DSO of buying energy from the market at times of peak electricity price), by technical reasons (in order to reduce overloading of network components during times of peak demand, or during periods when the system is weakened due to line/transformer maintenance or other outages).
The second service, DAPP, is designed to redistribute the power demand (load shifting) so that the constraints on the substations $s$ are fulfilled. This is done by computing, for each residential home $u$ connected to $s$, an individualised suggested power profile $P_{u}$ (i.e. different users may get different power profiles), so that if all users follow their power profile then the operational constraints on $s$, as suggested by EVT, are met. Each user $u$ is motivated to follow the suggested power profile $P_{u}$ by an individualised price policy based on $P_{u}$. Proposing individualised price policies avoids the problem of rebound peaks, i.e. where the demand peaks are simply shifted through the day, which may violate operational constraints.

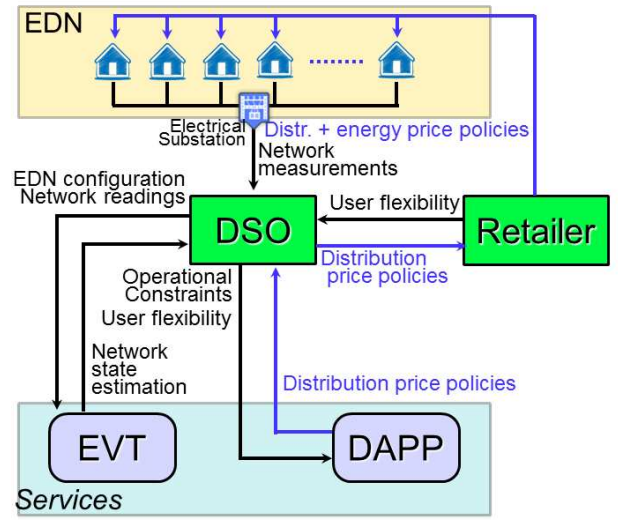

Fig. 2. The proposed services architecture.

\section{B. The DAPP Service}

In this section, the DAPP service is described. In order to understand how DAPP works, we first define how we model residential users flexibility (Section IV-B1). Then, we outline the DAPP service input-output behaviour (Section IV-B2). Third, we formally define one of the main requirements for the DAPP service, i.e., the fact that resulting individualised price policies must be non-discriminatory (Section IV-B3). Finally, we describe the algorithm underlying the DAPP service (Section IV-B4) and we prove that it outputs non-discriminatory price policies (Section IV-B5).

The notation used in the DAPP service is as follows: a timeslots set $T$ is a finite set of contiguous time-slots, all having the same duration $\tau$ (in minutes). Without loss of generality, we will assume $T$ to contain time-slot indices. A power profile is a function $P: T \rightarrow \mathbb{R}$, where we write $P^{t}$ for $P(t)$. A power profile $P_{1}$ follows a power profile $P_{2}$ if and only if $P_{1}(t) \leq P_{2}^{t}$ for all $t \in T$. The area of a power profile $P$ on $T$ is $\tau \sum_{t \in T} P^{t}$, i.e., the overall energy yielded by $P$. Finally, a Linear Programming $(L P)$ problem is a minimisation problem over a set of linear inequalities (constraints) on real variables.

1) Residential User Flexibility Model: In our approach, each residential user $u$ is provided, with a given periodicity (every day in our experiments), with an individualised price policy to be followed. Such price policy is defined on the basis of an individualised power profile $P_{u}$. The resulting tariff for $u$, which we call DAPP tariff, is based on two prices for energy, the high price and the low price: if user $u$ needs power $\tilde{d}_{u}^{t}$ in time-slot $t$, then $u$ will pay the low price if $\tilde{d}_{u}^{t} \leq P_{u}^{t}$, and 


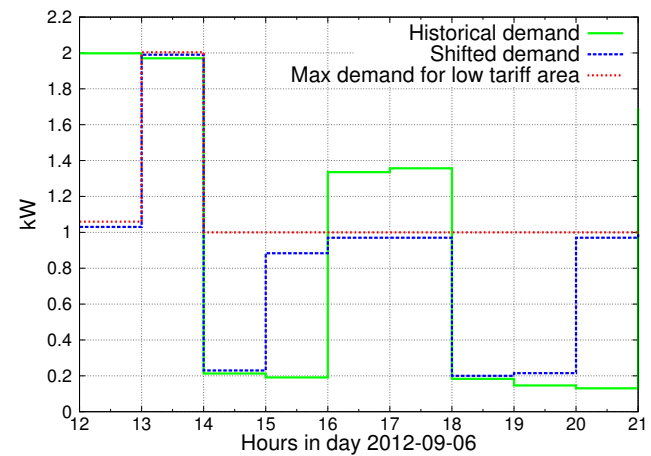

Fig. 3. Historical demand compared to power profile output by DAPP for a single home on a given day in our evaluation scenario.

the high tariff otherwise. As a consequence, we call low tariff area the area of $P_{u}$ on $T$. Note that the DAPP tariff is: (i) Inclining Block Rate (IBR) (two prices are used depending on user power demand); (ii) ToU ( $P_{u}$ varies with time); and (iii) individualised ( $P_{u}$ varies with the user too). As an example, Fig. 3 shows the individualised power profile $P_{u}$ (red curve) together with the actual power demand $d_{u}$ (green curve) on the 6th of September 2012 for a selected user $u$ connected to a selected substation $s$ in the reference scenario we will use for our experimental evaluation (see Section V). In the timeslot $t_{1}$ from 16:00 to 17:00, the user is outside the low tariff area (i.e., $d_{u}^{t_{1}}>P_{u}^{t_{1}}$ ), thus the high tariff is applied, whilst in the preceding time-slot $t_{2}$ the low tariff is applied. In order to stay inside the low tariff area also in $t_{1}$, the user should be flexible and, as an example, move approximately $0.3 \mathrm{~kW}$ of power demand (i.e., $d_{u}^{t_{1}}-P_{u}^{t_{1}}$ ) from $t_{1}$ to $t_{2}$ (load shifting).

In this section, we want to provide a mathematical model for user flexibility, based on the one in [14], in order to show the effectiveness of the DAPP-based methodology in scenarios in which the flexibility of each user may vary. To this aim, we proceed as follows. We model flexibility of a residential user $u$ by means of a load shifting capability. Such capability may be either "physical", i.e., user $u$ has a battery installed at home, or "virtual", i.e., user $u$ has to shift the loads of other appliances, in order to stay inside the low tariff area.

Given this, we model the load shifting capability, both in the physical and in the virtual case, as an ESS: the power rate of the ESS defines a bound on load shifting in each time-slot, whilst the capacity of the ESS gives a bound on the summation of consecutive load shifts. Namely, in our mathematical model, the flexibility of $u$ is a pair $\left(Q_{u}, R_{u}\right)$, where $Q_{u}$ is the ESS capacity (i.e., the maximum energy which may be stored, in $\mathrm{kWh}$ ) and $R_{u}$ is the ESS power rate (i.e., the maximum power in $\mathrm{kW}$ which may be used from or saved into the ESS in a given time-slot). In the example of Fig. 3, in order to perform the above described load shifting, it is sufficient to have $Q_{u}=$ $0.3 \mathrm{kWh}$ and $R_{u}=0.3 \mathrm{~kW}$. Since the user has to move $0.3 \mathrm{~kW}$ in one hour, from time-slot $t_{1}$ to time-slot $t_{2}$, the ESS must be charged by $0.3 \mathrm{~kW}$ in time-slot $t_{1}$ and discharged by $0.3 \mathrm{~kW}$ in time-slot $t_{2}$. Both actions require at least $Q_{u}=0.3 \mathrm{kWh}$ and $R_{u}=0.3 \mathrm{~kW}$. Of course, the ESS modelling the load shifting capability should have been charged during time-slot $t_{2}$ (from $15: 00$ to $16: 00$, where the user needs less energy than the one allowed in low tariff), and then discharged during time-slot $t_{1}$ (from 16:00 to 17:00, where instead the user needs more energy than the one allowed in low tariff). Instead, in order to be able to stay in the low tariff area of Fig. 3 for all the displayed 9 hours (from 12:00 to 21:00), the user flexibility required is $Q_{u}=1 \mathrm{kWh}$, with a power rate $R_{u}=1 \mathrm{~kW}$, since the user is $1 \mathrm{~kW}$ outside the low tariff area in the time-slot from 12:00 to 13:00. A possible shifted demand with respect to a (1 $\mathrm{kWh}, 1 \mathrm{~kW}$ ) flexibility, which is always inside the low tariff area, is shown in the blue curve in Fig. 3. In the following, we will call charge/discharge plan a power profile $a_{u}$ returning, for each time-slot $t$, the action taken on a ESS of capacity $Q_{u}$ and power rate $R_{u}$. Namely, if $a_{u}^{t} \geq 0$, then the ESS is charged by $a_{u}^{t} \mathrm{~kW}$ (in Fig. 3 this happens, e.g., from 15:00 to 16:00). Otherwise, if $a_{u}^{t}<0$, then the ESS is discharged by $a_{u}^{t} \mathrm{~kW}$ (in Fig. 3 this happens, e.g., from 12:00 to 13:00). Of course, if $a_{u}^{t}=0$ no loads are shifted (in Fig. 3 this happens, e.g., from 13:00 to 14:00).

2) DAPP Input and Output: We now describe in detail input and output for our DAPP algorithm (for a high-level view, see Fig. 4). Namely, DAPP requires the following input:

1) a set of users $U$ connected to a substation $s$;

2) a time-slots set $T$ (typically with a time span one day in the future);

3) the desired power profile $P_{s}$ (in $\mathrm{kW}$ ) on $T$ for the substation $s$, as decided by the DSO on the basis of EVT output;

4) three per-unit tariffs $b_{l} \leq b_{h}, \beta \in \mathbb{R}^{+}$coming from the energy retailer: respectively, the low (buy) price, the high (buy) price, and the sell price for energy;

5) for each $u \in U$, a forecast $d_{u}$ for the power profile of $u$ in $T$ (this may be computed on the basis of $\tilde{d}_{u}$, i.e., of the power profile of $u$ in the days preceding $T$ using, e.g., [28]);

6) for each user $u \in U$, the maximum power (in $\mathrm{kW}$ ) $C_{u} \in$ $\mathbb{R}$ supported by the home main, as defined in the energy contract for electricity consumption and production (e.g., 3 or $6 \mathrm{~kW}$ );

7) for each user $u \in U$, the flexibility of $u$ as a pair $\left(R_{u}, Q_{u}\right)$ (see Sect. IV-B1);

8) the minimum energy (in $\mathrm{kWh}$ ) that must be contained in the resulting low tariff area of each user $u$, as a coefficient $\alpha$ multiplying the user energy contract $C_{u}$. As an example, if $\alpha=2$ and the maximum power supported by home $u$ main is $6 \mathrm{~kW}$, then the low tariff area of $u$ must contain at least $12 \mathrm{kWh}$ on all period $T$. In our experiments, we always use $\alpha=1$.

The output of DAPP is a set of individualised power profiles $P_{u}$ on $T$, for each residential user $u \in U$. Note that each $P_{u}$ defines a low tariff area. Namely, the DAPP (output) tariff, for a given user $u$, is defined to incentivise the user to follow the output power profile region as follows: i) if $u$ is producing energy, then the sell price $\beta$ is applied; ii) if $u$ is consuming energy, then either the low price $b_{l}$ or the high price $b_{h}$ are applied, depending on the power profile of $u$ following $P_{u}$ or not, respectively.

3) Non-Discriminatory Price Policies: Intuitively, in order to have residential users actually agreeing on paying bills based on individual price policies, it is necessary that such 
price policies are non-discriminatory. This is also important for the DSO and/or the energy retailer, as releasing discriminatory price policies would decrease the number of users accepting the price schema. In our context, we informally state that a set of individualised price policies is non-discriminatory if and only if all residential users have the same opportunities to always pay the low tariff, i.e., if individualised price policies, in the same period of time, are all at the same relative distance from each user's habits. The proposed DAPP price policies are designed to always be non-discriminatory, even in the case that users do not perform load shifting in order to follow the suggested load profile (see the discussion on the robustness of the DAPP price policies in Section V-C).

We formally define this notion as follows. First of all, we define a tariff system $\mathcal{T}$ as a triple $\mathcal{T}=\left(b_{l}, b_{h}, \beta, \mathcal{P}\right)$, where $b_{l}, b_{h}, \beta \in \mathbb{R}^{+}$are, respectively, the low price and the high price for buying energy and the price for selling energy, as decided by the energy retailer, whilst $\mathcal{P}=\left\{P_{u} \mid u \in U\right\}$ is the set of individualised price policies. Since prices $b_{l}, b_{h}, \beta$ are equal for all residential users, they are non-discriminatory by construction. As for $\mathcal{P}$, the following holds. If the power demand of a user $u$, as a response to an individualised price policy $P_{u}$, is $\hat{d}_{u}^{t}$ on a given time-slot $t$, then $u$ will pay $b_{l} \min \left\{P_{u}^{t}, \hat{d}_{u}^{t}\right\}+b_{h} \max \left\{\hat{d}_{u}^{t}-P_{u}^{t}, 0\right\}$ (here, we do not consider the case in which $\hat{d}_{u}^{t}<0$, as production is always paid with the same price $\beta$ ). Since our goal is to show that all users must have the same opportunity to stay inside the low tariff area, we have to focus on $\max \left\{\hat{d}_{u}^{t}-P_{u}^{t}, 0\right\}$, which is the load shifting which is required by user $u$ in order to stay inside the low tariff area. Intuitively, if $P_{u}$ forces user $u$ to perform load shifting in the same way $P_{u^{\prime}}$ does for $u^{\prime}$ (for any pair $\left.u, u^{\prime} \in U\right)$, then $\mathcal{P}$ is non-discriminatory.

In order to formally define this concept, we define, for each user $u \in U$ and time-slot $t \in T$, the load shifting required by $u$ in $t$ as:

$$
\zeta_{u}^{t}=\frac{\max \left\{\hat{d}_{u}^{t}-P_{u}^{t}, 0\right\}}{\hat{d}_{u}^{t}}
$$

That is, the load shifting required by a user is the percentage of power (with respect to the historical demand) which must be shifted to keep the resulting demand inside the low tariff area. Given the definition of required load shifting, we say that a tariff system $\mathcal{T}$ is $r$-non-discriminatory if:

$$
\xi=\operatorname{stddev}_{u \in U} \operatorname{avg}_{t \in T} \zeta_{u}^{t} \leq r
$$

where, for any (finite) set $V$, we have

$$
\operatorname{avg}_{v \in V} \psi(v)=\mu_{\psi}=\frac{1}{|V|} \sum_{v \in V} \psi(v)
$$

and

$$
\operatorname{stddev}_{v \in V} \varphi(v)=\sqrt{\frac{1}{|V|} \sum_{v \in V}\left(\varphi(v)-\mu_{\varphi}\right)^{2}}
$$

As an example, $\mathcal{T}$ is 0.1 -non-discriminatory if the standard deviation of the set of averaged load shifts is below $10 \%$ (which is a reasonable threshold in our present paper). This

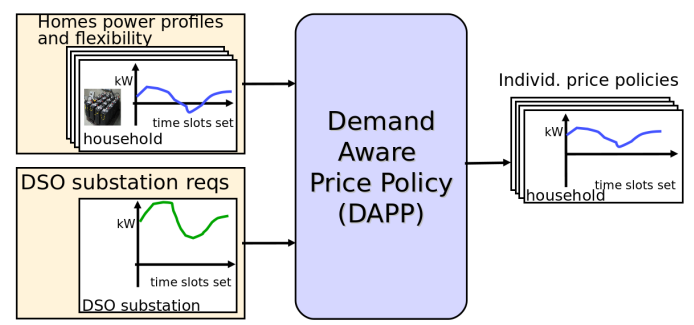

Fig. 4. DAPP input and output on a single DSO substation.

allows us to measure how much the DAPP price policies are non-discriminatory, including the case where users do not follow the suggested power profiles (see Section V-C).

Finally, we note that estimating the flexibility of each user is very important in order to enable DAPP to output nondiscriminatory price policies. To this aim, in our experiments, we compute the capacity of the flexibility of each residential user $u$ as the average on all power variations between consecutive time-slots in the historical power demand of $u$. As for power rate, we fix it as the typical power rate for ESSs, i.e., $2 \mathrm{~kW}$. That is, for all $u \in U$, if the power profile of $u$ from the available history $\tilde{T}$ is $\tilde{d}_{u}$, the flexibility of $u$ is defined as follows:

$$
\left(Q_{u}, R_{u}\right)=\left(\tau \operatorname{avg}_{t \in \tilde{T}}\left|\tilde{d}_{u}^{t+1}-\tilde{d}_{u}^{t}\right|, 2\right)
$$

In this way, we assume $u$ to be able to shift the demand of $u$ not more than $u$ already does in the historical records.

4) DAPP Algorithm: DAPP algorithm consists in the following steps:

1) set up LP problem $L$;

2) solve $L$ via an LP solver (CPLEX in our case);

3 ) extract the required output from the solution of $L$.

In the following, we describe the mathematical formulation of the LP problem $L$. To this aim, first of all we list all $3|U||T|+|T|$ decision variables involved.

- For each user $u \in U$ and time-slot $t \in T$, a decision variable $P_{u}^{t}$, modelling the upper bound (in $\mathrm{kW}$ ) of the low tariff area of user $u$ in time-slot $t$.

- For each user $u \in U$ and time-slot $t \in T$, a decision variable $b_{u}^{t}$, modelling the state of charge (in $\mathrm{kWh}$ ) of the load shifting capability of user $u$ in time-slot $t$.

- For each user $u \in U$ and time-slot $t \in T$, a decision variable $a_{u}^{t}$, modelling a charge/discharge plan, i.e., the charge (if positive) or the discharge (if negative) action (in $\mathrm{kW}$ ) decided by user $u$ in time-slot $t$ in order to stay within the low tariff area. Note that, in our problem formulation, we only consider fixed load shifting capabilities, and do not try to compute the charging/discharging of "mobile" battery appliances such as PEVs. In our experiments, we consider recharging of PEVs as an additional load (that is, residential homes with a Plugin Electric Vehicle(s) (PEV) have higher power demand).

- For each time-slot $t \in T$, a decision variable $\Delta^{t}$, modelling the aggregated user demand (in $\mathrm{kW}$ ) which exceeds substation desired power profile in $t$. 
Then, we describe all $2|U|+2|T|+6|U||T|$ constraints in $L$, involving the above defined decision variables and input values described in Section IV-B2.

$$
\begin{aligned}
& \text { Minimise } \sum_{t \in T} \Delta^{t} \\
& b_{u}^{t_{1}}=b_{u}^{t_{|T|}}=\frac{Q_{u}}{2}: \quad \forall u \in U \\
& b_{u}^{t+1}=b_{u}^{t}+\frac{\tau}{60} a_{u}^{t} \quad: \quad \forall u \in U, t \in T \\
& a_{u}^{t} \geq \min \left\{-d_{u}^{t}, 0\right\} \quad: \quad \forall u \in U, t \in T \\
& a_{u}^{t}+d_{u}^{t} \leq P_{u}^{t} \quad: \quad \forall u \in U, t \in T \\
& \sum_{t \in T} P_{u}^{t} \geq \frac{60}{\tau} \alpha C_{u} \quad: \quad \forall u \in U \\
& \sum_{u \in U} P_{u}^{t} \leq P_{s}^{t}+\Delta^{t} \quad: \quad \forall t \in T \\
& 0 \leq P_{u}^{t} \leq C_{u} \quad: \quad \forall u \in U, t \in T \\
& 0 \leq b_{u}^{t} \leq Q_{u} \quad: \quad \forall u \in U, t \in T \\
&-R_{u} \leq a_{u}^{t} \leq R_{u} \quad: \quad \forall u \in U, t \in T \\
& \Delta^{t} \geq 0 \quad: \quad \forall t \in T
\end{aligned}
$$

- Constraint (7): for each user $u \in U$, the state of charge at the beginning of $T$ and at the end of $T$ must be both equal to half of user flexibility.

- Constraint (8): for each user $u \in U$ and time-slot $t \in T$, the ESS modelling flexibility of $u$ behaves like a battery, i.e., the action taken at time-slot $t$ effects state of charge at time $t+1$.

- Constraint (9): for each user $u \in U$ and time-slot $t \in T$, the ESS modelling flexibility of $u$ cannot be used to inject power into the grid, i.e., it may be discharged only up to the current power consumption.

- Constraint (10): for each user $u \in U$ and time-slot $t \in T$, the power resulting from applying the ESS action to the current demand must be below the price policy upper bound. This constraint ensures that DAPP will output non-discriminatory price policies (see Section IV-B3). This is formally proved in Proposition 1.

- Constraint (11): for each user $u \in U$, the resulting low tariff area for $u$ must contain at least $\alpha C_{u} \mathrm{kWh}$ on all $T$. This avoids reduction of the overall demand volume.

- Constraint (12) and objective function (6): for each timeslot $t \in T$, if all users synchronize and use the maximum energy allowed in their low tariff areas, the resulting aggregated demand must be below the substation desired threshold, plus an offset to be minimised (in order to achieve peak shaving).

- Finally, the remaining constraints (13)-(16) define the upper and lower bounds for each decision variable. Note that, since we minimise $\sum_{t \in T} \Delta^{t}$, we simply require each $\Delta^{t}$ to be non-negative.

The final output of DAPP is obtained by retrieving the values of $P_{u}^{t}$ from the solution returned by the LP solver. Note that the solution returned by the LP solver also contains a charge/discharge plan for each user $u \in U$ (using values of $a_{u}^{t}$ decision variables). This allows us to define, for each user $u \in U$, the DAPP collaborative power profile of $u c_{u}: T \rightarrow \mathbb{R}$ as the result of applying the charge/discharge plan $a_{u}$ to the input forecasted profile $d_{u}$, i.e., for all $t \in T$

$$
c_{u}^{t}=d_{u}^{t}+a_{u}^{t}
$$

Note that each collaborative power profile is always inside the low tariff area defined by Constraint 10 .

5) DAPP Output Price Policies Are Non-Discriminatory: In this section, we formally prove that the individualised price policies output by our DAPP service are indeed nondiscriminatory as wanted, if the forecasting is precise.

Proposition 1. Let $\tilde{d}_{u}^{t}$ be the historical power demand of user $u \in U$ in time-slot $t \in T$, and let $d_{u}^{t}$ be the forecast of $\tilde{d}_{u}^{t}$ given in input to DAPP. If the forecasting error is 0 , i.e., $d_{u}^{t}-\tilde{d}_{u}^{t}=0$, then the price policies output by DAPP are $0-$ non-discriminatory.

Proof. If the relative forecasting error is 0 then, for each user $u \in U, d_{u}^{t}=\tilde{d}_{u}^{t}$. Let us assume that, for each user $u \in U$, the collaborative power profile $c_{u}$ output by DAPP is the actual response of $u$ to the individualised price policy $P_{u}$ output by DAPP for $u$. Hence, $\hat{d}_{u}^{t}=c_{u}^{t}=d_{u}^{t}+a_{u}^{t}$ for all $t \in T, u \in U$. By Constraint 10, we have that $\hat{d}_{u}^{t} \leq P_{u}^{t}$, thus $\operatorname{avg}_{t \in T} \zeta_{u}^{t}=0$ for all $u \in U$ which implies $\xi=\operatorname{stddev}_{u \in U} \operatorname{avg}_{t \in T} \zeta_{u}^{t} \leq r$ for each $r \geq 0$.

Note that, in the general case, for a given user $u \in U$, $c_{u}$ is not the only possible choice for $\hat{d}_{u}$ in order to always stay inside the low tariff area of $u$. However, the proof given above works for any $\hat{d}_{u}=c_{u}^{\prime} \neq c_{u}$, provided that $c_{u}^{t^{\prime}}=$ $d_{u}^{t}+a_{u}^{t^{\prime}}$ and that $a_{u}{ }^{\prime}$ fulfils Constraints (8), (9), (10), (14) and (15). In particular, since Constraint (10) is satisfied, the thesis holds.

As for the case in which the forecasting error is nonzero, Section V-C shows the results of applying (2) to our experimental scenarios, by considering the worst case in which the users do not respond to the individualised price policies (i.e., $\hat{d}_{u}^{t}=\tilde{d}_{u}^{t}$ for all $u \in U, t \in T$ ). This will show that the output of DAPP is robust with respect to errors in the input forecasted demand. In the proposed scheme, users are motivated to stay inside the low tariff area by the low energy price provided by DAPP individualised price policies. It should be noted that DAPP does not directly provide an incentive to users to increase their flexibility, e.g., by installing ESS. However, users with high flexibility play an important role in enabling load shifting, which in turn allows DAPP to return a smaller value for the upper bound $\left(P_{u}\right)$ for the low tariff area of such users. Hence, the aggregated user demand which exceeds the substation desired power profile will be smaller (i.e., the value of the objective function of the LP problem described in Section IV-B4 is smaller). Since this is a benefit for the DSO, it will be in the DSOs interest to provide an economic incentive for users willing to increase their flexibility.

\section{Implementation Issues}

In order to implement the DAPP load shifting, it was assumed that each user's battery and/or controllable appliances 
are controlled automatically using a home energy management system, whose objective is to minimise the user's energy bill, by staying inside the low tariff area as much as possible. In the SmartHG project [1] (on which the presented paper is based), a test-bed was developed where residential users had Home Area Networks (HANs) with load sensors and actuators and a corresponding communication infrastructure. This provided a means of implementing the individualised price policies. The SmartHG test bed and communication infrastructure are outlined in more detail in [29]. A full evaluation of this home energy management system is out of the scope of this paper, which is devoted to computational services at the network level.

However, the individualised pricing schemes proposed in this paper could in theoretically be implemented using any home energy management system, such as those already proposed the literature (e.g. [12], [13]), or through any combination of direct load control of home appliances, or indirect user responses to price signals. One of the advantages of the proposed approach is that the price signal user sees is very simple: in any given hour receive either the low or the high tariff. However, the proposed demand management approach is likely to be more effective in cases where there is a home energy management system, which can automatically implement the demand response, rather than depending on an indirect or manual user response to the price signal. The communication hardware cost of implementing individualised price policies is expected to be low, since it is assumed that all users already have smart meters installed, and users can receive individual rather than global prices without any hardware modifications.

\section{CAse Study and Results}

\section{A. Base Case}

The case study network used in this paper is taken from the European Commission project "SmartHG" [1]. This network is a suburban/rural 10kV system with a weakly-meshed structure. A reduced version of the network schematic diagram is shown in Fig. 5. The network has a peak demand of 3.2 MW (with the largest demands occurring in winter), and serves approximately 1,600 customers. There are $46 \mathrm{MV}$ nodes in the network, where each MV node corresponds to a secondary transformer substation $(10: 0.4 \mathrm{kV})$. Of these 46 secondary substations, 30 serve suburban/rural residential customers $(77 \%$ of the total network demand), and the remaining $16 \mathrm{MV}$ substations serve demand comprising of factory, district heating and water pumping loads. It was assumed that price policies outlined in Section IV only affect the residential demands. Measurements of power consumption were recorded at each substation at hourly intervals over the course of a two year period from September 2012 to September 2014, in the form of aggregated smart meter measurements. In addition, smart meter data was available from selected individual users in the network.

This data was used to create the reference scenario called "Base Case" which shows the results calculated using the actual recorded data from the network for the two-year period from September 2012 to September 2014. The results

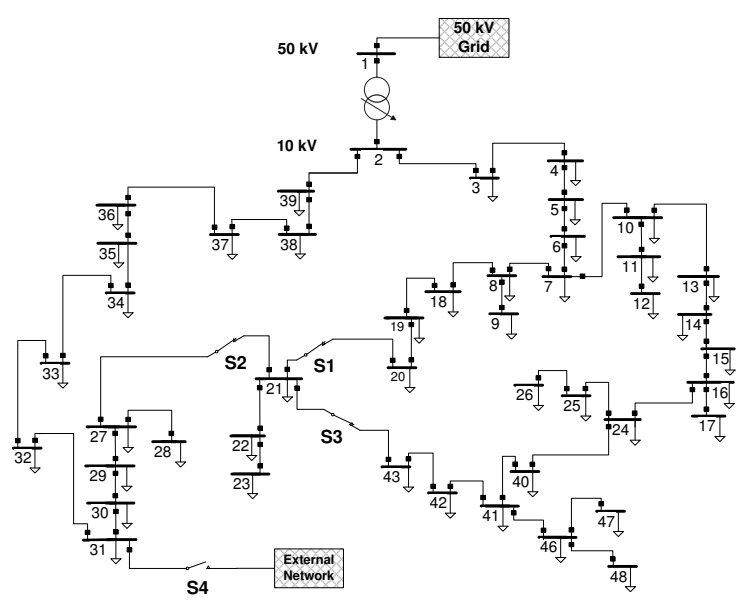

Fig. 5. Schematic diagram of MV distribution network case study.

calculated using the actual recorded data from the network for the two-year period from September 2012 to September 2014. All users received a fixed (i.e., flat) electricity price during this period.

\section{B. Test Scenarios}

The test scenarios described below and in Table I are extensions of the Base Case. Scenarios 1 and 2 show the results of simulations designed to examine the effects of both "global" and "individualised" price policies:

- Scenario 1a All residential users in the case study network receive the same ToU price designed to shift demand away from the peak hours (e.g. a "global" price policy). Households do not have energy storage or PEV.

- Scenario 1b All residential users receive the individualised ToU price policy proposed by the DAPP service. Households do not have energy storage or PEV.

- Scenario 2a All users receive the same "global" ToU price policy designed to shift demand away from the peak hours. 50\% of the households (randomly-selected) have PEV (with $16 \mathrm{kWh}$ capacity and $13 \mathrm{~kW}$ power rate). The PEV data used in this study was taken from actual vehicle charging data from the "Test-an-EV" project [30].

- Scenario 2b All residential users receive the individualised ToU price policy proposed by the DAPP service. $50 \%$ of the households have a PEV as in Scenario 2a, and all households are equipped with energy storage in the form of a PowerWall battery [31] (with $7 \mathrm{kWh}$ capacity and $2 \mathrm{~kW}$ power rate).

TABLE I

SCENARIO CHARACTERISTICS SUMMARY

\begin{tabular}{cccc}
\hline Scenario & Price policy & PEV & ESS \\
\hline Base Case & flat rate & No & No \\
1a & global & No & No \\
$\mathbf{1 b}$ & indiv & No & No \\
$\mathbf{2 a}$ & global & $50 \%$ & $100 \%$ \\
$\mathbf{2 b}$ & indiv & $50 \%$ & $100 \%$ \\
\hline
\end{tabular}




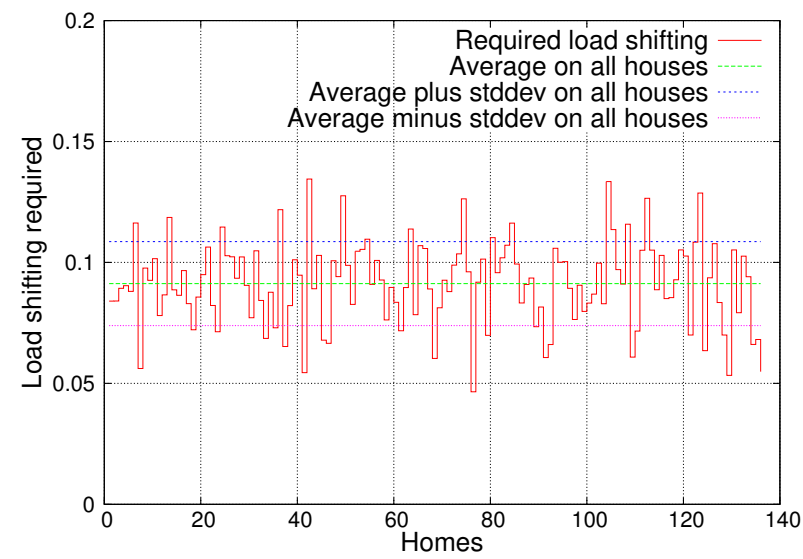

Fig. 6. Load shifing required by users connected to the substation with most connected houses in Scenario $1 \mathrm{~b}$.

\section{Robustness of Non-Discrimination in DAPP Price Policies}

In this section we show that the individualised price policies output by DAPP for each residential user are indeed nondiscriminatory (see Section IV-B3) in a robust way. Proposition 1 only holds if the forecast has no errors. In real applications, there will always be an error in power forecasting, thus we want to show that the price policies output by DAPP are $r$-non-discriminatory with a very low $r$, e.g., $r=0.1$. To this aim, we compute $\xi$ from (2) with $\hat{d}_{u}^{t}=\tilde{d}_{u}^{t}$, i.e., when there is no response to the individualised price policies (which implies that the response coincides with the historical demand). This allows us to investigate the worst case scenario and to show that, even if each user does not perform load shifting at all (i.e., $a_{u}^{t}=0$ for all $u \in U, t \in T$ ), the price policies output by DAPP are 0.1 -non-discriminatory. This proves robustness of DAPP output price policies, as they are not strongly dependent on the power demand forecasting.

Considering Scenarios $1 \mathrm{~b}$ and $2 \mathrm{~b}$ (the only ones involving DAPP output), we obtain the following results for the nondiscrimination index $\xi$ in (2):

Scenario $1 \mathrm{~b}: 2.08 \%$;

Scenario $2 b: 5.32 \%$

As a result, we have that in both our scenarios the individualised price policies output by DAPP are indeed 0.1 non-discriminatory (and thus non-discriminatory), as required. Finally, in order to show an interesting example of load shifting requirements, Fig. 6 depicts the load shifting required by all 136 houses connected to the substation with highest number of connected users in our Scenario 1b. Moreover, Fig. 6 also shows the mean of the load shifting of all 136 houses, and the standard deviation from this mean. As a result, we have that the standard deviation of the required load shifting on these 136 houses is less than $2 \%$. On the other hand, the standard deviation $\sigma$ of the total energy demand (i.e., accumulated on the whole period of two years) for the 136 houses in this example is $22 \%$ of the maximum total energy demand. This shows that, even in a scenario in which power demands have an high variance among users, the price policies output by DAPP are $0.02-$ non-discriminatory.
D. Simulated Impacts of Price Policies on Network Demand Profiles and Load Factor

1) Impact on Demand Profiles: This section shows the impact on load profiles at each substation calculated by simulation of each of the above scenarios. Figures $7 \mathrm{a}$ and $7 \mathrm{~b}$ show a sample of the load profiles for the Base Case, Scenario 1a and Scenario 1b. These are shown for the Winter Peak and Summer Minimum demand cases respectively.

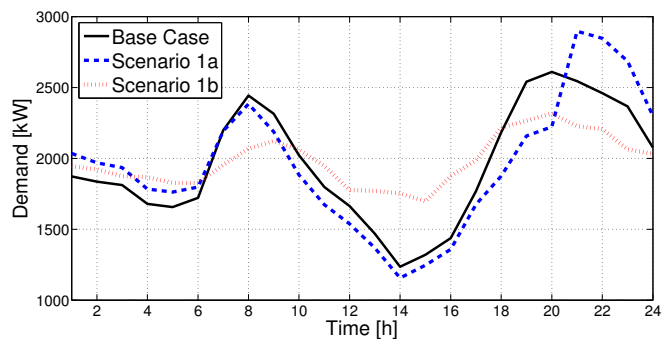

(a)

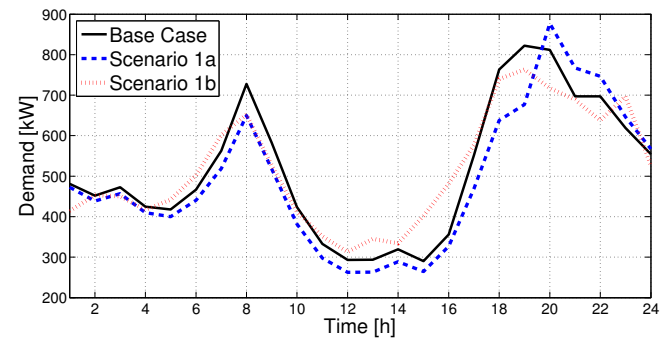

(b)

Fig. 7. Scenario 1 - Time series of aggregate residential demand profiles showing Base Case, global price policy (Scenario 1a), and individualised price policy (Scenario 1b) for: a) Winter Peak; b) Summer Minimum.

It can be seen from Fig. 7 that the global price policy (dashed line, Scenario 1a) results in a rebound demand peak similar to that recorded in Fig. 1, Section I and [16]. For the individual price policy case (dotted line, Scenario 1b, Fig. 7b), the demand peaks are much reduced due to the effect of the DAPP algorithm. This significantly improves the load factor (the ratio of average to maximum load).

For Scenario 2 (Fig. 8), the overall demand is increased due to the influence of PEV load, with larger peaks in the evening hours. The global price policy results show a large demand rebound at hours 20:00 - 22:00 (dashed lines, Scenario 2a). The proposed individual price policy results in a much flatter demand profile (dotted lines, Scenario $2 b$ in Fig. 7b).

2) Impact on Aggregate Load Factor: The aggregate load factors, calculated across all residential customers, are provided in Tables II and III. These were calculated for typical "Winter Peak" and "Summer Minimum" days, and also for the "Overall" case, which calculates the total load factor over the two years of the simulation.

These results reflect the pattern shown in Fig. 1, Section III and in the load profile results in Figs. 7-8. It can be seen in that the global price policy (Scenarios 1a and 2a) increases the overall magnitude of the demand peaks and reduces the demand middle of the day, which produces lower load factors than in the Base Case. In contrast, the individualised price policy flattens the demand profiles and increases load factors 


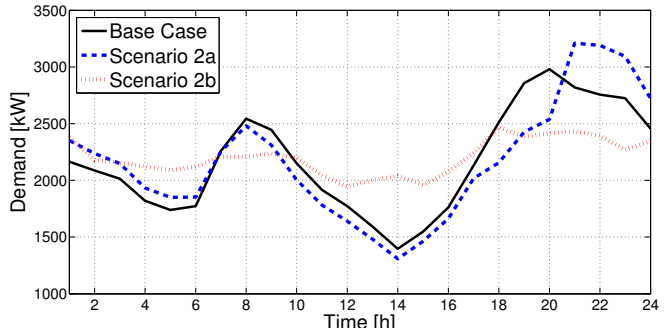

(a)

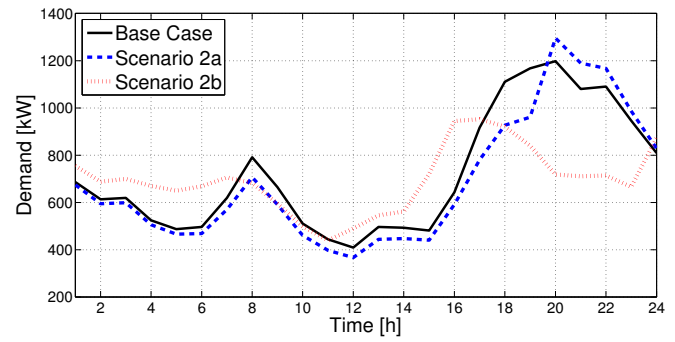

(b)

Fig. 8. Scenario 2 - Time series of aggregate residential demand profiles showing Base Case, global price policy (Scenario 2a), and individualised price policy (Scenario 2b) for: a) Winter Peak; b) Summer Minimum.

(Scenarios $1 b$ and $2 b$ ).

TABLE II

SCENARIO 1 AgGREgATEd LOAD FACTORS

\begin{tabular}{cccc}
\hline Load Factor & Base Case & Scenario 1a & Scenario 1b \\
\hline Winter Peak & 0.7054 & 0.6164 & 0.8017 \\
Summer Min & 0.6205 & 0.5794 & 0.6257 \\
Overall & 0.3327 & 0.2897 & 0.3933 \\
\hline
\end{tabular}

The load factors in the "Overall" case (final rows of Tables II and III are much lower than the Winter Peak/Summer Minimum day cases, since the "Overall" values represent the average load factor calculated over the entire two year period, considering all of the seasonal variations during this time.

The results in Tables II and III clearly show the potential benefits of applying the proposed individualised pricing scheme. In Scenario 1 there is an overall annual load factor improvement compared to the Base Case by using DAPP of $15.4 \%$, and in Scenario 2, the corresponding figure is $27.2 \%$. It is also shown that the amount of load flattening varies throughout the course of the year, and in the Summer Minimum day in Scenario 1, the improvement in load factor compared to the Base Case is not significant (less than $1 \%$ ). However, in Scenario $2 b$, the corresponding load factor improvement is $18.7 \%$. This suggests that the benefits of applying an individualised are likely to be much more significant in networks with PEV and ESS.

This simulated increase in load factors due to the application of individualised price policies would have clear benefits for the DSO. This would reduce the amount of energy to be purchased from the wholesale market during expensive peak hours, and the flatter load profiles would result in less instances where network is overloaded, potentially reducing network maintenance and upgrade costs and allowing deferral
TABLE III

SCENARIO 2 AGgREGATED LOAD FACTORS

\begin{tabular}{cccc}
\hline Load Factor & Base Case & Scenario 2a & Scenario 2b \\
\hline Winter Peak & 0.7116 & 0.6176 & 0.8124 \\
Summer Min & 0.5927 & 0.5280 & 0.7038 \\
Overall & 0.3471 & 0.3084 & 0.4415 \\
\hline
\end{tabular}

of network investments. In the following section, some of the impacts of the proposed price policies on the distribution network operation are examined in more detail.

\section{E. Simulated Network Impacts of Price Policies}

1) Impact on Network Power Flows: The impacts on power flows throughout the MV network is shown in Fig. 9 for Scenarios 1 and 2, using the maximum thermal MVA flow expressed as a percentage of the line rating. The thermal MVA flow is calculated for all of lines and transformers in the MV distribution network (Fig. 5), and the maximum value for each day in the 2-year period is shown. From both Figs. 9a and 9b, it is clear that the global price policy (Scenario 1a and 2a, dashed lines) results in heavier line loading values, whereas the individualised price policy (Scenario $1 \mathrm{~b}$ and $2 \mathrm{~b}$, dotted lines) results in reduced line loading. This result is expected, since it is shown in Section V-D2 that the individualised price policy reduces the magnitude of the demand peaks and improves the load factor. Table IV shows the results for the number of overloads (MVA flow $>100 \%$ of line rating) in each case.

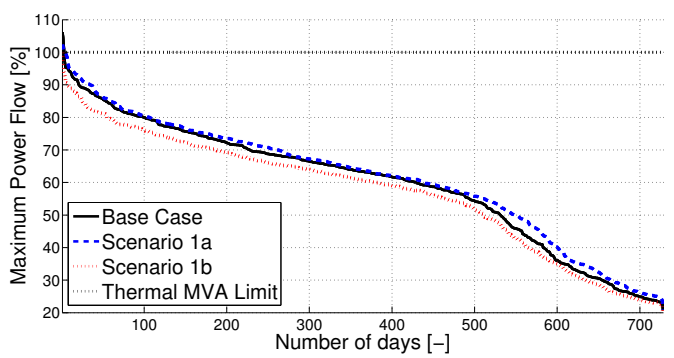

(a)

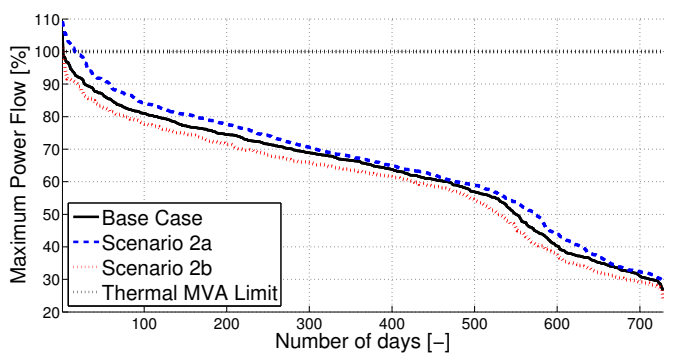

(b)

Fig. 9. Daily maximum loading as a percentage of the line limits for: a) Scenario 1; b) Scenario 2.

2) Impact on Network Voltages: The number of low voltage violations in the distribution network case study are summarised in Table V. Any voltages lower than 0.97 p.u. ${ }^{3}$ were

${ }^{3} \mathrm{MV}$ network voltage limits are set tighter than the typical statutory voltage limits of $1.1-0.9$ p.u., since it is expected that there will be significant further voltage drops on the LV feeders downstream of the MV substations in Fig. 5 (LV feeders are not shown in the schematic), particularly along longer lines. 
TABLE IV

SUMMARY OF LINE THERMAL MVA LIMIT VIOLATIONS

\begin{tabular}{cccc}
\hline $\begin{array}{c}\text { No. Overloads } \\
(>100 \%)\end{array}$ & Base Case & $\begin{array}{c}\text { Global } \\
\text { Price Policy }\end{array}$ & $\begin{array}{c}\text { Individual } \\
\text { Price Policy }\end{array}$ \\
\hline Scenario 1 & 3 & 6 & 0 \\
Scenario 2 & 3 & 18 & 2 \\
\hline
\end{tabular}

recorded as voltage violations. High voltage events are not considered in the study, nor are events involving network faults and planned/unplanned outages, which would affect the voltage profiles. These results show that several voltage violations occur in both the Base Case and Scenario 1a and 2a (global price policy) cases. These low voltages occur during time of peak loading, in the parts of the network with the greatest electrical distance from the primary substation. In the individual price policy simulations, Scenario $1 b$ and $2 b$, there were no violations, due to the reductions in peak loading.

TABLE V

SUMMARY OF LOW VOLTAGE Violations

\begin{tabular}{cccc}
\hline $\begin{array}{c}\text { No. Low Voltages } \\
(>0.97 \text { p.u. })\end{array}$ & Base Case & $\begin{array}{c}\text { Global } \\
\text { Price Policy }\end{array}$ & $\begin{array}{c}\text { Individual } \\
\text { Price Policy }\end{array}$ \\
\hline Scenario 1 & 2 & 2 & 0 \\
Scenario 2 & 2 & 2 & 0 \\
\hline
\end{tabular}

3) Impact on Network Line Losses: The analysis of the total losses in the MV test case network showed some differences in network losses as a results of applying the global and individualised price policy scenarios:

- Scenario 1: Typical cumulative losses in the MV network are $142 \mathrm{MWh} /$ year and $68 \mathrm{MVAr} / \mathrm{year}$. The individualised price policy produced $1.5-1.6 \%$ lower line losses compared to the global price policy case.

- Scenario 2: Typical cumulative losses in the MV network $162 \mathrm{MWh} / \mathrm{year}$ and $78 \mathrm{MVAr} / \mathrm{year}$. The individualised price policy had $2.2-2.3 \%$ lower line losses.

\section{CONCLUSIONS}

This paper proposed a novel approach to DSM, using an "individualised" price policy, which incentivises users to optimally manage flexible demands. The primary objective is to reduce peaks in the overall distribution system demand, in such a way that the average price received by each end user is non-discriminatory. This approach is compared to traditional "global" price policy schemes, where all users (or a large number of users in the same network region or associated with the same retailer) are given the same price policy. The analysis in the paper illustrates the potential advantages of the individualised price policy approach through extensive simulations using a case study of residential users in a typical European distribution network, where each of the scenarios used was based on actual data from network end-users.

It was demonstrated both in the trial results in [16] and in the results in Section V that the use of global DSM price policies can cause synchronisation of user demand patterns, reducing load diversity and creating undesirable "rebound" effects. The individualised price policy approach proposed in this paper has advantages over a global policy approach. It was shown that the individualised price policy can increase the load factor, and improve voltage and line loading conditions, and reduce network losses, compared to a global DSM price signal.

The results here are based on trials from a Danish DSO and extensive simulations using recorded demand data. However, the actual behaviour of a particular group of customers in response to a price signal (global or individualised) is complex and difficult to predict accurately. Therefore it is not possible to draw general conclusions, which apply to other users across all regions and market conditions, based on these results. Despite this, the presented results are informative in that they illustrate that there are significant problems in using a global price policy approach for residential DSM, and show that the proposed individualised price policy approach is able to resolve these problems and provide operational benefits for the DSO. There are other potential long-term DSO benefits which are not assessed in this paper, such as network investment deferral, and improvement of equipment lifetimes due to reduced instances of overloading. One limitation of the approach is that it does not specifically encourage users to increase their flexibility. The DSO may wish to offer economic compensation to encourage users to install more ESS and increase their flexibility, since this flexibility clearly benefits the DSO in terms of reducing operational and network investment costs. A possible approach to providing these incentives would be to allocate a portion of the projected savings from the deferral of network investments for this purpose.

\section{ACKNOWLEDGEMENTS}

The authors would like to thank Federico Mari and Ivano Salvo of Sapienza University of Rome for their contribution in the development of the DAPP service, Jorn Klaas Gruber of IMDEA Energy for his contribution to the design of the EVT service, and Lars Elmegaard of SEAS-NVE for providing the data from the case study distribution system.

\section{REFERENCES}

[1] European Commision project SmartHG. (2016, May). [Online]. Available: www.smarthg.eu

[2] E. Lannoye, D. Flynn, and M. O'Malley, "Evaluation of power system flexibility," IEEE Trans. Power Syst., vol. 2, pp. 922-931, 2012.

[3] D. Crossley, "Assessment and development of network driven demand side management measures," Interational Energy Agency, Tech. Rep. Task XV Research Report No 2, Oct. 2008.

[4] Red Electrica de Espana. (2016, May) Red Electrica de Espana Interruptibility Service. [Online]. Available: http://www.ree.es/en/activities/operation-of-the-electricitysystem/interruptibility-service

[5] National Grid. (2015) National grid balancing services. [Online]. Available: http://www2.nationalgrid.com/uk/services/balancing-services/

[6] J. Torriti, "Price-based demand side management: Assessing the impacts of time-of-use tariffs on residential electricity demand and peak shifting in northern italy," Energy, vol. 44, pp. 576 - 583, 2012.

[7] P. Samadi, H. Mohsenian-Rad, V. Wong, and R. Schober, "Tackling the load uncertainty challenges for energy consumption scheduling in smart grid," IEEE Trans. Smart Grid, vol. 4, no. 2, pp. 1007-1016, June 2013.

[8] B. Hayes, I. Hernando-Gil, A. Collin, G. Harrison, and S. Djokic, "Optimal power flow for maximizing network benefits from demand-side management," IEEE Trans. Power Syst., vol. 29, no. 4, pp. 1739-1747, July 2014. 
[9] C. Adika and L. Wang, "Demand-side bidding strategy for residential energy management in a smart grid environment," IEEE Trans. Smart Grid, vol. 5, no. 4, pp. 1724-1733, July 2014.

[10] T. Mancini, F. Mari, I. Melatti, I. Salvo, E. Tronci, J. Gruber, B. Hayes, M. Prodanovic, and L. Elmegaard, "Demand-aware price policy synthesis and verification services for smart grids," in IEEE International Conference on Smart Grid Communications (SmartGridComm), Nov 2014, pp. 794-799.

[11] Y. Li, B. L. Ng, M. Trayer, and L. Liu, "Automated residential demand response: Algorithmic implications of pricing models," IEEE Trans. Smart Grid, vol. 3, no. 4, pp. 1712-1721, Dec 2012.

[12] Y. Ozturk, D. Senthilkumar, S. Kumar, and G. Lee, "An intelligent home energy management system to improve demand response," IEEE Trans. Smart Grid, vol. 4, no. 2, pp. 694-701, June 2013.

[13] Z. Zhao, W. C. Lee, Y. Shin, and K.-B. Song, "An optimal power scheduling method for demand response in home energy management system," IEEE Trans. Smart Grid, vol. 4, no. 3, pp. 1391-1400, Sept 2013.

[14] T. Mancini, F. Mari, I. Melatti, I. Salvo, E. Tronci, J. K. Gruber, B. Hayes, M. Prodanovic, and L. Elmegaard, "User flexibility aware price policy synthesis for smart grids," in Proc. of IEEE DSD 2015, 2015.

[15] Irish Social Science Data Archive. (2016, May) CER Smart Metering Project website. [Online]. Available: http://www.ucd.ie/issda/data/commissionforenergyregulationcer/

[16] SEAS-NVE. (2016, May) Win with new electricity habits. [Online]. Available: http://ipaper.ipapercms.dk/SeasNVE/Winwithnewelectricityhabits/

[17] J. Medina, N. Muller, and I. Roytelman, "Demand response and distribution grid operations: Opportunities and challenges," IEEE Trans. Smart Grid, vol. 1, pp. $193-198,2010$

[18] D. Pudjianto, C. Ramsay, and G. Strbac, "Virtual power plant and system integration of distributed energy resources," IET Renewable Power Generation, vol. 1, pp. $10-16,2007$.

[19] N. Ruiz, I. Cobelo, and J. Oyarzabal, "A direct load control model for virtual power plant management," IEEE Trans. on Power Syst., vol. 24 pp. $959-966,2009$.

[20] B. Moradzadeh, "Optimal distribution reconfiguration and demand management within practical operational constraints," Ph.D. dissertation, University of Tennessee, 2013.

[21] J. F. Hauer and J. E. Dagle, "Grid of the future white paper on review of recent reliability issues and system events," Consortium for Electric Reliability Technology Solutions, Tech. Rep., 1999.

[22] D. Livengood and R. Larson, "The energy box: Locally automated optimal control of residential electricity usage," Service Science, vol. 1, no. 1 , pp. 1-16, 2009

[23] M. Roozbehani, M. Dahleh, and S. Mitter, "On the stability of wholesale electricity markets under real-time pricing," in Decision and Control (CDC), 2010 49th IEEE Conference on, Dec 2010, pp. 1911-1918.

[24] L. Pieltain Fernandez, T. Roman, R. Cossent, C. Domingo, and P. Friias, "Assessment of the impact of plug-in electric vehicles on distribution networks," IEEE Trans. on Power Syst., vol. 26, no. 1, pp. 206-213, 2011.

[25] S. Shafiee, M. Fotuhi-Firuzabad, and M. Rastegar, "Investigating the impacts of plug-in hybrid electric vehicles on power distribution systems," IEEE Trans. Smart Grid, vol. 4, no. 3, pp. 1351-1360, Sept 2013.

[26] B. Hayes, J. Gruber, and M. Prodanovic, "A closed-loop state estimation tool for MV network monitoring and operation," IEEE Trans. Smart Grid, vol. 6, no. 4, pp. 2116-2125, 2015.

[27] B. P. Hayes and M. Prodanovic, "State forecasting and operational planning for distribution network energy management systems," IEEE Transactions on Smart Grid, vol. 7, no. 2, pp. 1002-1011, March 2016.

[28] L. de Castro and P. Cramton, "Prediction markets for electricity demand," in Communication, Control, and Computing (Allerton), 2012 50th Annual Allerton Conference on, Oct 2012, pp. 1097-1104.

[29] V. Alimguzhin, F. Mari, I. Melatti, E. Tronci, E. Ebeid, S. Mikkelsen, R. Hylsberg Jacobsen, J. Gruber, B. Hayes, F. Huerta, and M. Prodanovic, "A glimpse of smarthg project test-bed and communication infrastructure," in Digital System Design (DSD), 2015 Euromicro Conference on, Aug 2015, pp. 225-232.

[30] Test-an-EV project. (2016, May) Electrical Vehicle (EV) Data. [Online]. Available: http://smarthg.di.uniroma1.it/Test-an-EV/

[31] Tesla Motors. (2016, May) Tesla PowerWall website. [Online]. Available: https://www.teslamotors.com/powerwall

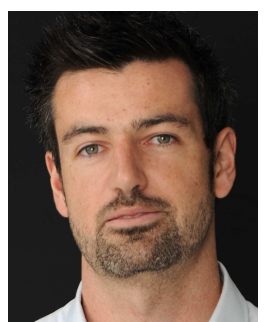

Barry Hayes (S'09, M'12) received the B. Eng. degree in electrical engineering from University College Cork, Cork, Ireland in 2005, the M. Eng. from the National University of Ireland, Maynooth, Ireland in 2008, and the Ph.D degree from the University of Edinburgh, Edinburgh, UK in 2013. He worked in industry at Intel near Dublin, Ireland from 2005-2009 and was a visiting researcher at National Grid UK in 2011 and at the University of Tennessee, TN, U.S.A. in 2016. He is currently a Marie Curie research fellow at Institute IMDEA Energy, Madrid, Spain. His research interests include the network integration of renewable energy sources, and the operation and planning of future power systems.

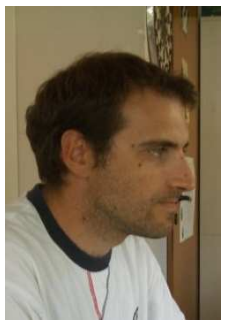

Igor Melatti is an assistant professor at the Computer Science Department of Sapienza University of Rome, Rome, Italy. His current research interests comprise: formal methods, automatic verification algorithms, model checking, software verification, hybrid systems, automatic synthesis of reactive programs from formal specification, systems biology, smart grids.

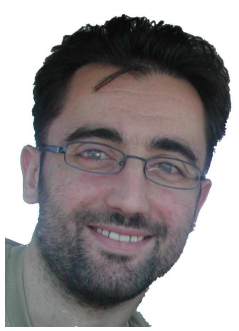

Toni Mancini has a Ph.D. in Computer Science Engineering and is assistant professor at the Computer Science Department of Sapienza University of Rome, Rome, Italy. His research interests comprise: artificial intelligence, formal verification, cyber-physical systems, control software synthesis, systems biology, smart grids.

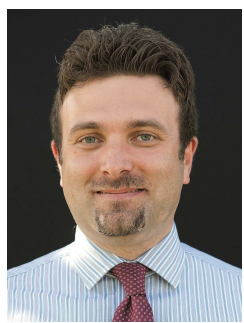

Milan Prodanovic (M01) received the B.Sc. degree in electrical engineering from the University of Belgrade, Serbia, in 1996 and the Ph.D. degree from Imperial College, London, U.K., in 2004. From 1997 to 1999 he was engaged with GVS engineering company, Serbia, developing UPS systems. From 1999 until 2010 he was a research associate in Electrical and Electronic Engineering at Imperial College, London, UK. Currently he is a Senior Researcher and Head of the Electrical Systems Unit at Institute IMDEA Energy, Madrid, Spain. His research interests include design and control of power electronics interfaces for distributed generation, micro-grids control and active management of distribution networks.

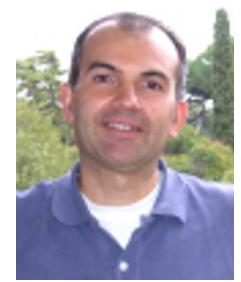

Enrico Tronci is an associate professor at the Computer Science Department of Sapienza University of Rome, Rome Italy. He received a masters degree in Electrical Engineering from Sapienza University of Rome and a Ph.D. degree in Applied Mathematics from Carnegie Mellon University. His research interests comprise: formal verification, model checking, system level formal verification, hybrid systems, embedded systems, cyber-physical systems, control software synthesis, smart grids, autonomous demand and response systems for smart grids, systems biol- 